\title{
Dispensierrecht in Deutschland
}

\author{
Bei den deutschen Hausärzten wird immer intensiver über die Medikamenten- \\ abgabe direkt aus der Praxis diskutiert. Nach dem Vortrag des Kollegen Ernst Gähler \\ aus Herisau über die Medikamentendispensation in der Schweiz hat diese Diskus- \\ sion noch einmal Fahrt aufgenommen.
}

Ulrich Weigeldt

Bundesvorsitzender Deutscher Hausärzteverband e.V. Facharzt für Allgemeinmedizin und Sportmedizin

In Deutschland werden Impfstoffe über den Sprechstundenbedarf direkt aus der Praxis abgegeben bzw. verabreicht. Obwohl hier durch das Einhalten einer Kühlkette nicht unerheblicher Aufwand besteht, gibt es keinerlei Klagen über diese Dispensierung von Medikamenten. In den Fokus genommen wurden dann jedoch die Verhältnisse im Notfalldienst und bei Hausbesuchen. Dabei sind wir Hausärzte nicht damit zufrieden, lediglich mit Ärztemustern ausgestattet agieren zu können, denn dies kann im Einzelfall auch rechtliche Probleme aufwerfen. Es besteht ein hohes Interesse daran, hier zu einer adäquaten Lösung zu kommen. Diese kann eigentlich nur darin bestehen, dass ein Medikamentensortiment in der Praxis zur Verfügung steht, das für die Versorgung der Patienten im Notdienst oder bei einem Hausbesuch sofort und sicher abgegeben werden kann.

\section{Derzeit werden die rechtlichen Rahmenbedingungen geprüft, um eine Selbstdispensation zu ermöglichen.}

Korrespondenz: Ulrich Weigeldt Deutscher Hausärzteverband e.V. Bleibtreustrasse 24 D-10707 Berlin hausaerzteverband.de
Das erhöht die Patientensicherheit, da sich Verordnung und Behandlung in einem Schritt vollziehen lassen und nicht ein Weg in die Apotheke des Patienten und/oder - falls verfügbar - der pflegenden oder betreuenden Angehörigen oder Freunde/ Nachbarn vorgeschaltet wird (Anmerkung: Wäre die Mobilität nicht gestört, bräuchte es ja keinen Hausbesuch). Zu Zeiten, in denen der Notdienst verrichtet wird, gibt es in der Regel auch nur die Notdienst habende Apotheke, also ist gerade in diesem Fall die Dispensation aus der Praxis respektive der Arzttasche sinnvoll und für die schnelle Behandlung speziell im Notfall ideal.

Wir müssen davon ausgehen, dass insbesondere auf dem Land, wo die Apothekendichte ebenso abnimmt wie die Arztdichte, die Versorgung der Patienten schneller, besser und sicherer gestaltet werden kann, wenn Medikamente direkt aus der Praxis abgegeben werden können. Ob dies einen Umfang

\section{DEUTSCHER}

\section{HAUSÄRZTEVERBAND}

in dem von den Apotheken gefürchteten Ausmass annehmen wird, ist ausserordentlich fraglich. Es ist nicht zu erwarten, dass das gesamte in einer OffizinApotheke vorgehaltene Arzneimittelspektrum in der Praxis eines Hausarztes vorgehalten werden muss oder wird, und wir wissen zudem, dass der Apothekenumsatz nicht nur durch rezeptierte Medikamente erzeugt wird, um es vorsichtig auszudrücken. Wir können uns im Gegenteil auch in der Frage der Selbstdispensation sehr gut eine Zusammenarbeit mit Apotheken und Apothekern vorstellen.

Derzeit werden die rechtlichen Rahmenbedingungen geprüft, um eine Umsetzung der Selbstdispensation zu ermöglichen. Berücksichtigt man den Trend zu IT-gestützten Verfahren im Gesundheitswesen - Stichwort elektronisches Rezept - zur Reduzierung von Bürokratie und Zeitaufwand, wird man an einer Medikamentenabgabe aus der Praxis kaum vorbeikommen. Damit kann und soll kein Zwang ausgeübt werden, und wir gehen immer davon aus, dass die Entscheidungsfreiheit bei den Patienten liegen muss, auch in diesem Fall. Wir wissen aus den freien Verträgen mit Krankenversicherungen wie der AOK Baden-Württemberg, dass die Patienten die angebotenen Optionen gerne nutzen, sich freiwillig für die Teilnahme an der Hausarztzentrierten Versorgung entscheiden und dann dabei bleiben. Warum soll man diesen Patienten nicht auch die Möglichkeit einräumen, ihr Medikament direkt bei der Behandlung von ihrem Hausarzt ausgehändigt zu bekommen? Dann wird man sehen, wie viele Menschen von dieser Möglichkeit Gebrauch machen und wie viele ihre Medikamente lieber aus ihrer Apotheke beziehen wollen.

Wir haben nicht, wie Sie in der Schweiz, das Instrument der Volksabstimmung, aber wir können den Patienten Wahlmöglichkeiten anbieten, und sie werden entscheiden. Wir wollen sie weder dazu zwingen, Medikamente nur beim Hausarzt zu erhalten, noch dazu, eine mitunter ja auch entfernte Apotheke aufsuchen zu müssen. 